\title{
Enabling Progress Towards Life Detection on NASA Missions
}

$A$ White Paper from the Network for Life Detection

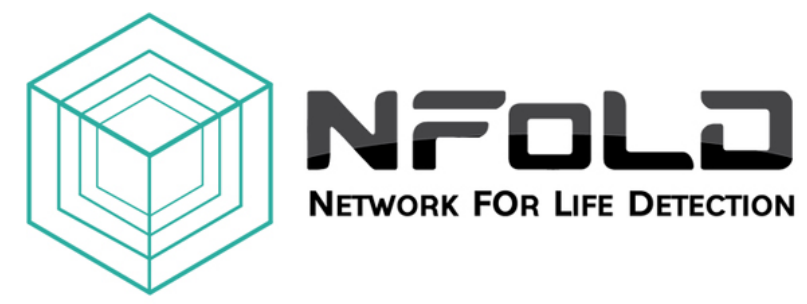

Submitted by

NfoLD Co-Leads:

Britney Schmidt, Georgia Tech, corresponding author (britneys@eas.gatech.edu, 404-385-1869); Sarah Stewart Johnson, Georgetown University; Tori Hoehler, NASA Ames; Heather Graham, NASA Goddard; Jeff Bowman, UCSD; Sanjoy Som, Blue Marble Space Institute of Science;

NfoLD Steering Committee Co-Authors:

Laura Barge, JPL; Nathalie Cabrol, SETI; Alex Pavlov, NASA Goddard; Alexandra Pontefract, Georgetown University; Amanda Stockton, Georgia Tech; Beth Orcutt, Bigelow; Brook Nunn, University of Washington; Christine Foreman, Montana State University; David Stillman, Southwest Research Institute; Everett Shock, Arizona State University; Fabien Kenig, University of Illinois, Chicago; Gordon Love, UC Riverside; Kristin Bergmann, MIT: Pablo Sobron, SETI; Richard Mathies, Berkeley; Roland Hatzenpichler, Montana State University; Shashan Yu, NASA JPL; Wesley Swingley, Northern Illinois University; Dan Jones, New Mexico Inst. Mining \& Tech.

Early Career Co-authors: Justin Lawrence, Frances Bryson, Elizabeth Spiers, Chase Chivers, Taylor Plattner, Andrew Mullen, Ashley Hanna, Georgia Tech; Jacob Buffo, Dartmouth;

Community Endorsers: Shawn Domagal-Goldman, NASA Goddard; Karyn Rogers, RPI; Giada Arney, NASA Goddard; Victoria Meadows, U Washington; Jennifer Glass, Georgia Tech; Peter Doran, LSU; Alison Olcott, U Kansas; Kris Zacny, Honeybee Robotics; Graham Lau, BMSIS; Peter Willis, JPL; Tyler Mackey, UNM; Natalie Grefenstette, Santa Fe Inst./BMSIS; Luoth Chou, NASA Goddard/Georgetown U.; Jennifer Blank, NASA Ames/BMSIS; Gareth Trubl, LLNL; Alta Howells, ASU; Joseph Pasterski, U Illinois Chicago; Morgan Cable, JPL; Shane Byrne, U Az. Robert Dziak, NOAA; Bryanna Henderson, JPL; Dale Winebrenner, $U$ Washington; Anne Dekas, Stanford; Alison Murray, DRI; Brent Christner, U Florida; Connor Nixon, NASA Goddard; Michael Malaska, JPL; Alexis Templeton, University of Colorado; Evan Sneed, Penn State U; Mariam Naseem, Space Generation Advisory Counci; Craig Everroad, NASA ARC; Niki Parenteau, NASA ARC; Emily Felder, Pasadena City College, Kaitlin Rempfert, U CO Boulder; Margaret Weng, Georgetown U; Emily Kraus, CO Mines; Maëva Millan NASA GSFC / Georgetown U; Tristan Caro, U. CO Boulder; Vinayak Shastri, University of St Andrews; Clark Kevin, NASA ARC; Brandi Kiel Reese, Dauphin Island Sea Lab; Marjorie Cantine, Massachusetts Institute of Technology. 


\section{Enabling Progress Towards Life Detection on NASA Missions}

The quest for life elsewhere in the solar system is the next frontier in NASA science: the search for life is expressed as among the top five goals of MEPAG, OPAG, VeXAG and SBAG for the next decade, demonstrating how aligned the planetary science community is with this pursuit. The possibility of detecting evidence of life within planetary environments can be within our grasp if a balance of near-term investments and programmatic changes are implemented this decade, alongside a plan to prepare promising new technologies for missions.

In this white paper, we discuss a number of significant obstacles regarding how astrobiology is translated into planetary exploration, and propose solutions. We focus on the solar system, recognizing that the search for life on exoplanets is more directly enabled by the progress of telescope technology and is covered as part of the ongoing 2020 Astrophysics Decadal Survey. We outline key approaches developed in the past decade of astrobiology research that are promising for both short- and long-term implementation, review mission-ready technology for advancing life detection in the decade 2023-2032, and advocate for advancements that can be achieved in ten years to enable life detection in 2033 and beyond. We also discuss programmatic changes that can help compelling life detection missions be selected.

\section{Summary of Recommendations}

To advance toward life detection requires meaningful integration of astrobiology objectives, expertise, and measurement technology into NASA missions. To foster such integration, we recommend that NASA:

1) Engage the astrobiology science and technology communities in developing (i) a standard framework in which the traceability of specific measurements to life detection objectives can be assessed relative to a set of universally applied criteria; and (ii) a curated set of reference materials that provide a common, rather than self-defined, basis for demonstrating instrument performance with respect to life detection objectives.

2) Develop and implement a comprehensive plan for advancing the readiness of technology required to support life detection objectives: (i) Establish standing programs in the mold of, e.g., ICEE and ICEE-2, that provide early stage funding to a range of novel measurement approaches in order to 'buy down risk' early and identify promising candidates for maturation. (ii) Maintain a well-funded technology maturation program that is specific to life detection objectives. The program should comprise an end-to-end scope that encompasses sample handling/processing, "front-end" systems, detection, and onboard data processing.

3) Consider changes to how proposals are reviewed and how risk posture is established for mission and instrument selection, and consider further mission studies of end-to-end life detection missions, in order to support the planetary science and astrobiology communities in pursuing life detection concepts within all mission classes.

\section{Relationship to 2017 NASEM Report on Astrobiology Strategy for the Search for Life} Our recommendations echo the findings of the 2017 NASEM report on Astrobiology Strategy as regards universal biosignatures (p.68), risk posture (p.92), science drivers for sample handling (p.92) technology development (p.93), and the need for better integration of astrobiology expertise into the mission arc (p.93). Those findings should be included in this decadal survey.

\section{Barriers in Pursuit of Life Detection}

Life detection faces a barrier of perception: that it must be an all-in, flagship-level commitment that supplants the mainstream objectives of planetary sciences and, if 
"unsuccessful", dooms future spacecraft missions and in particular, life detection efforts. On the contrary, however, it can be a systematic endeavor that utilizes multiple mission classes, to grow our understanding of planetary environments at every step along their evolutionary paths. Environmental characterization is not just possible, but essential, for life detection: context is critical for interpreting either positive or negative results and important for planning follow up observations, for example as described in the 2017 Europa Lander Study (Hand et al 2017). The past decades of astrobiology research demonstrate that life detection objectives extend, rather than supplant, the goals of planetary science; this decadal survey report can do much to shift perceptions by articulating this message and paving the way.

A second barrier lies in the institutional and cultural challenges inherent in fully integrating astrobiology expertise and technology into the conception, development, and implementation of missions. The traditional objectives of planetary exploration are served effectively by a welldefined community, body of knowledge, and suite of measurement approaches that developed around and thus are fundamentally organized around missions and NASA programmatic activities. But the answers to our loftiest questions requires that we expand beyond the current sphere of planetary sciences. Much of the expertise and technology needed to address our highest science goals lies distributed across a diversity of disciplines that have little historical connection to missions and, in many cases, have not perceived their relevance to solar system exploration. The intricacies of a mission arc can seem impenetrable and inaccessible to the very communities whose knowledge is most relevant to the pursuit of life detection objectives. By the same token, detailed understanding of the subject matter, language, and methodology associated with life detection may seem no more tractable to the leaders of planetary sciences - the people who generally conceive, decide upon, and implement missions, including those that will seek evidence of life. The need for better integration spans both science and technology associated with life detection but, in the context of flight, is most acute in instrument development.

"Astrobiological exploration in particular is severely limited by a lack of flight-ready instruments that can address key questions regarding past or present life elsewhere in the Solar System. The committee recommends that a broad-based, sustained program of science instrument technology development be undertaken, and that this development include new instrument concepts as well as improvements in existing instruments. This instrument technology program should include the funding of development through TRL 6 for those instruments with the highest potential for making new discoveries." - Visions \& Voyages Decadal Survey

Despite this call for action, such challenges remain and are the primary focus of this white paper.

\section{Challenges}

The range of potential life detection measurements and approaches is large, diverse, and in many cases novel relative to those traditionally utilized in planetary exploration. This creates a challenging development path for life detection instruments and the missions that will fly them.

Measurement approaches that are a good fit for life detection objectives may lie outside the expertise of the traditional pool of reviewers in planetary science. Complexity and novelty in measurement approach can make it harder it to define Level 1 objectives that are comprehensible to the non-expert. This is not unique to life detection missions, but plagues instruments across many facets of exploration: cameras are intuitive to understand for visual beings, whereas plasmas, fields, chemical measurements, etc. may be more abstract to non-experts. However, the existence of a relatively well-defined set of "go to" measurement approaches in planetary sciences has enabled those in the field to develop a working understanding sufficient to serve as 
a basis for review. Moreover, the formal training typical in the planetary sciences community -geology, mineralogy, particles and fields, etc - inspired and/or grew from the measurements and instruments on missions thus far. This will not be true for many life detection approaches. Moreover, individual life detection objectives have potential to be addressed by a range of orthogonal approaches, the capabilities of which are conveyed in very different terms of detection limit, measurement quality, and reproducibility, against self-defined standards. These factors increase the chance that the specific expertise required to evaluate and compare the capabilities of differing approaches with respect to specific life detection objectives may not be represented on review teams such that the perceived risk of these techniques may be increased.

Relative to traditional planetary instrumentation, life detection instruments may have specific additional requirements that increase the end-to-end complexity of analysis. Rather than remote sensing or direct sample ingestion that is characteristic of most current approaches in planetary sciences, a range of life detection approaches will require sample processing that may include phase change, filtration, or chemical alteration (e.g., de-salting, derivatization). Many of these needs may be held in common among different approaches and would benefit from early development and standardization of approach. Despite their clear value, however, sample handling systems in open competition with science instruments may be undervalued by review panels, preventing them from receiving critical early development. In some cases, the potential complexity of both the information sought and the applicable background will necessitate "frontend" approaches (e.g., chemical or physical separation) that again add to instrument complexity. Finally, some approaches may require data-intensive measurements that would require onboard data processing that has yet to be developed. Collectively, the requisite complexity of many measurement approaches suggests that investment in life detection technology should embrace an end-to-end scope that enables not just measurements, but sample processing, front-end capability, and onboard data reduction.

Some of the approaches that may prove most relevant in pursuit of life detection will lack opportunities to develop the flight heritage that allows them to be considered "low risk" in cost or TRL. The general utility of many of the approaches traditionally utilized in planetary sciences means they can be flown to a range of targets and in a range of applications, thereby establishing flight heritage. In contrast, some life detection techniques may only be applicable in the context of a life detection mission and not, e.g., in flights to LEO, Moon, or small bodies. Such approaches will face the challenge of being proposed for the first time in the context of an expectedly high-profile mission.

Finally, confidence in any announcement of life detection will be bolstered by multiple lines of evidence that derive from independent measurements (see, e.g., Hand et al 2017).

Because of the inherent ambiguity in many known bio signatures, and the necessity of making multiple measurements on a sample, in situ detection of life is best advanced by integrated suites of instruments. -2017 NASEM

Instruments on flagship missions are competed separately, which limits the extent to which independent measurement approaches can be tailored for complementarity as a suite, unless proposed "together" as a suite from the outset. The ideally co-dependent nature of life detection measurements could also challenge the selection of complex approaches that, by virtue of novelty, are less well understood than the traditional planetary instrumentation. While PI-led missions can propose integrated suites, these missions have lower budgets and generally lower cost and technical risk in order to be selected, such that new approaches to life detection (or other scientific approaches) are less likely to be included. 


\section{Recommendations Explained}

Standardization in Life Detection Measurements

We strongly recommend that NASA engage the astrobiology science and technology communities in developing

(i) a standard framework in which the traceability of specific measurements to life detection objectives can be assessed relative to a set of universally applied criteria; and

(ii) a curated set of reference materials that provide a common, rather than self-defined, basis for demonstrating instrument performance with respect to life detection objectives. An end-to-end standardization that is agreed upon by the community at large will enable proposers to more clearly demonstrate traceability and performance with respect to life detection objectives, and promote concrete, apples-to-apples assessment of the capabilities of diverse approaches. The "Ladder of Life Detection" (LoLD; Neveu et al., 2018) represents a first effort to standardize the criteria on which the utility of different measurements is assessed, with its authors' stated intent being to initiate further dialog. Such a formulation could be implemented in the mission review process. Clear next steps are to engage the broader community in debating and finalizing a set of universally-applicable criteria, amass and organize the body of knowledge required to establish traceability between any evaluative process and the complete set of evidence that supports it, and provide a basis for assessing the availability and TRL of technologies that could support specific measurement objectives (see submission from The Center for Life Detection).

\section{Fostering Technology Development}

We recommend that NASA develop and implement a comprehensive plan for advancing the readiness of technology required to support life detection objectives: In accordance with the high priority of life detection objectives and the critical need for instrument development (as identified in Visions \& Voyages), we suggest that the life detection instrumentation program represent 50\% of $\mathrm{NASA}$ 's planned investment in planetary instrument development over the next decade. Specifically:

(i) Maintain a well-funded technology maturation program, similar to MATISSE and PICASSO, that is specific to life detection objectives. Such a program would be similar to the former ASTID program, and would guarantee that progress is made given the high value of life detection instrumentation and the low readiness of many of the most promising technologies. The program should comprise an end-to-end scope that also invests in sample handling and processing, "front-end" systems, detection, and onboard data processing/reduction. An important goal of this program should be to broaden the range of tools and techniques that could be brought to bear in a search for life. This should encompass both new approaches for measuring well understood indicators of life, as well as approaches that respond to emerging work on new biosignatures, for example complexity-based and "universal" biosignatures (see submission by Chou, Grefenstette, et al.). Recognizing the down-stream need for integration and common use among the range of elements supported in such a program, steps should be taken to provide baseline sample handling and instrument integration pathways to proposers from early in the development cycle, for instance at the TRL 4 level. For example, NASA centers and FFRDCs could be funded to work with instrument teams that successfully reach TRL 4 in order to help the instruments reach TRL 6 and be integrable into planned architectures, while preserving the science and contributions of the PI/instrument developers. This would enable more instruments to be developed in mission-relevant context and reduce technical risk. 
(ii) When preparing for flagship missions, continue programs like ICEE and ICEE-2 that provide early funding to a range of instruments in order to 'buy down risk' early and enable communication between the instrument team and the mission team. Such programs are effective ways to tailor instruments to challenging mission requirements/accommodation. Such early investments help prevent cost growth and increase the pool of viable instruments.

\section{Exploring Life Detection Mission Options}

We recommend that NASA support the planetary sciences and astrobiology communities in developing life detection mission concepts within all mission classes. The current system by which missions and instruments are planned and evaluated has potential to bias decisions against critical but new instruments, creating science risk. Yet early generations of NASA missions flew new or "unproven" instruments with success. Life detection can be a systematic endeavor that utilizes multiple mission classes and grows our understanding of planetary environments at every step. Moreover, the desirability of multiple independent lines of evidence for life and/or to place findings within environmental context, argues for the value of payloads that are enabled as a suite. In addition to technology investments, supporting a suite of mission concept studies, such as those supported for the present decadal survey, would tap the creativity of the community in conceiving novel concepts for life detection missions, and support the development of those concepts in a systems-engineering context. Ideally, the timing of such support should be such that a range of mature concepts can be proposed to the next New Frontiers opportunity.

(i) Consider changes to how proposals are reviewed and how risk posture is established for mission and instrument selection, and consider further mission studies of end-to-end life detection missions, in order to support the planetary science and astrobiology communities in pursuing life detection concepts within all mission classes.

Table 1: Example technologies with potential relevance to life detection objectives, their states of development, and traceability to the existing Ladder of Life Detection (LoLD). This table is meant to be illustrative, not complete. ${ }^{a}$ Estimated TRL for flight. TRL (6) denotes relevant systems designed for/operational on Earth. ${ }^{b}$ Relevant LoLD "rung": 0-Habitability, 1 Biofabrics, 2 - Potential metabolic byproducts, 3 - Potential biomolecule components, 4 Molecules \& Structures Conferring Function, 5 - Metabolism, 6 - Growth \& Reproduction, 7 Darwinian Evolution. '( )' indicates partial relevance. 'Italics-instrument selected, not yet flown

\begin{tabular}{|c|c|c|c|c|c|c|}
\hline Instrument & $\mathrm{TRL}^{\mathrm{a}}$ & LOLI $^{b}$ & Bench & Field & Flight ${ }^{\mathrm{C}}$ & Environ \\
\hline \multicolumn{7}{|c|}{ Spectroscopy } \\
\hline Infrared & 9 & $0,(5)$ & & & SAM TLS $^{1}$ & Surface \\
\hline \multirow[t]{2}{*}{ Raman } & 6 & \multirow[t]{3}{*}{0,2} & & & $R L S^{2}$ & Surface \\
\hline & $5-(6)$ & & & $\mathrm{MMRS}^{3}$ & & Surface \\
\hline Time-gated Raman & $4-9$ & & $\mathrm{SUCR}^{4}$ & & SuperCam ${ }^{5}$ & Surface \\
\hline SERS/SERRS & 4 & $0,2,3,5$ & Tang et al $(2016)^{6}$ & & & Surface \\
\hline UV Resonance Raman & $8-9$ & $0,2,3,4$ & & & SHERLOC ${ }^{7}$ & Surface \\
\hline SRS & $2-4$ & $0,2,5,(6)$ & Hu et al $(2019)^{8}$ & & & Surface \\
\hline Raman+LIBS & $4-5$ & \multirow[t]{2}{*}{0,2} & & InVADER $^{9}$ & & Ocean \\
\hline SSE & $4-(6)$ & & CIRS $^{10}$ & & & Surface \\
\hline
\end{tabular}




\begin{tabular}{|c|c|c|c|c|c|c|}
\hline \multirow[t]{2}{*}{ UV Fluorescence } & $2-4$ & \multirow{4}{*}{1,4} & $\mathrm{CoCoBi}^{11}$ & & & Surface \\
\hline & \multirow[t]{3}{*}{$3-4$} & & C-LIFE ${ }^{12}$ & & & Surface \\
\hline $\begin{array}{l}\text { UV Fluorescence, IR } \\
\text { Imaging }\end{array}$ & & & OWL-I ${ }^{13}$ & & & Surface \\
\hline VSWIR Spectroscopy & & & ELSSIE $^{14}$ & & & Surface \\
\hline Protein Fluorescence & $4-(5)$ & 3 & & $\mathrm{PFS}^{15}$ & & Ocean \\
\hline \multicolumn{7}{|c|}{ Mass Spectrometry and Separations } \\
\hline \multirow[t]{2}{*}{ GC/LD-Ion Trap-MS } & 8 & \multirow[t]{5}{*}{$0,2,3,5$} & & & $M O M A^{16}$ & Surface \\
\hline & $3-4$ & & & & $\operatorname{DraMS} S^{17}$ & Surface \\
\hline \multirow[t]{2}{*}{ GC-MS } & 9 & & & & $\begin{array}{c}\text { Huygens } \\
\text { NMS }^{18}\end{array}$ & Surface \\
\hline & 9 & & & & MSL SAM $^{1}$ & Surface \\
\hline GCxGC-MS & $3-4$ & & $\begin{array}{l}\text { MASPEX- } \\
\text { ORCA }^{19}\end{array}$ & & & Surface \\
\hline \multirow[t]{2}{*}{ TOF-MS } & $3-4$ & \multirow[t]{5}{*}{$0,2,3,5$} & ELF-ENIJA $^{20}$ & & & Orbital \\
\hline & $3-4$ & & ELF-MASPEX $^{20}$ & & & Orbital \\
\hline Cycloidal-MS & (6) & & & NEREUS/TETHYS ${ }^{21}$ & & Ocean \\
\hline Q-MS & (6) & & & ISMS $^{21}$ & & Ocean \\
\hline Ion Trap-MS & $4-(6)$ & & & DOMS/MiniDOMS $^{21}$ & & Ocean \\
\hline Orbitrap-MS & $3-4$ & $0,2,3,5$ & CORALS $^{22}$ & & & Surface \\
\hline CESI-MS/LIF/C4D & $3-4$ & \multirow[t]{4}{*}{$0,2,3,4,5$} & OCEANS $^{23}$ & & & Surface \\
\hline \multirow[t]{2}{*}{ CE-LIF } & $3-4$ & & $\mathrm{EOA}^{24}$ & & & Orbital \\
\hline & $3-4$ & & $\mathrm{MOAB}^{25}$ & & & Surface \\
\hline ME-LIF & 6 & & & Chemical Laptop ${ }^{26}$ & & Surface \\
\hline $\mathrm{LC}$ & $5-(6)$ & $0,2,3$ & & ISLC $^{27}$ & & Ocean \\
\hline LC-TOF-MS & $3-4$ & $0,2,3,5$ & OASIS $^{28}$ & & & Surface \\
\hline LC-MS/SFC-MS & 4 & $0,2,3$ & SFE-SFC $^{29}$ & & & Surface \\
\hline \multicolumn{7}{|c|}{ Electrochemistry } \\
\hline Hydrogel ISEs & 9 & \multirow[t]{2}{*}{0,5} & & & $\begin{array}{l}\text { MECA- } \\
\text { WCL }^{30}\end{array}$ & Surface \\
\hline Microfluidic SC-ISEs & $3-4$ & & $\mathrm{MICA}^{31}$ & & & Surface \\
\hline \multicolumn{7}{|c|}{ Microscopy } \\
\hline Optical Microscopy & 9 & \multirow[t]{4}{*}{1,6} & & & MECA-OM ${ }^{32}$ & Surface \\
\hline \multirow[t]{3}{*}{ Holographic Microscopy } & $3-(6)$ & & & SHAMU $^{33}$ & & Ocean \\
\hline & 4 & & & Lensless $^{34}$ & & Ocean \\
\hline & $3-5$ & & & Submersible DHM $^{35}$ & & Ocean \\
\hline \multirow[t]{2}{*}{ Fluorescence Microscopy } & $3-4$ & \multirow[t]{3}{*}{$1,4,6$} & 3D FLFM ${ }^{36}$ & & & Ocean \\
\hline & 3 & & Deep UV $\mathrm{UV}^{37}$ & & & Surface \\
\hline Microspectroscopy & 9 & & & & CIVA-M $^{38}$ & Surface \\
\hline
\end{tabular}




\begin{tabular}{|c|c|c|c|c|c|c|}
\hline $\begin{array}{l}\text { Atomic Force } \\
\text { Microscopy }\end{array}$ & 9 & \multirow[t]{2}{*}{1,6} & & & $\begin{array}{l}\text { MECA- } \\
\text { AFM }^{32}\end{array}$ & Surface \\
\hline Electron Microscopy & 4 & & $\mathrm{mSEM}^{39}$ & & & Surface \\
\hline Flow Cytometry & $3-4$ & 1,4 & Fluorescence $\mathrm{FC}^{40}$ & & & Surface \\
\hline \multicolumn{7}{|c|}{ Informational Polymer Detection } \\
\hline $\mathrm{D} / \mathrm{R} / \mathrm{XNA}$ sequencer & 4 & \multirow[t]{2}{*}{3,4} & & SETG $^{41}$ & & Surface \\
\hline qPCR, DNA/antibody & $5-(6)$ & & & $3 \mathrm{G}-\mathrm{ESP}^{42}$ & & Ocean \\
\hline \multicolumn{7}{|c|}{ Complementary Instrumentation Suites } \\
\hline Impact ionization $+\mathrm{QMS}$ & 9 & \multirow[t]{2}{*}{0} & & & $\mathrm{CDA}_{4}^{43} ; \mathrm{INMS}^{4}$ & Orbital \\
\hline $\begin{array}{c}\text { Impact } \\
\text { ionization+GCMS }\end{array}$ & 8 & & & & $\begin{array}{l}S U D A^{45} \\
M A S P E X^{46}\end{array}$ & Orbital \\
\hline
\end{tabular}

Text References: Hand et al 2017, Report of the Europa Lander SDT; Neveu et al 2018, Astrobio. 18:111375-1402. Table References: [1] Mahaffy, P. R. et al. (2012) Space Sci. Rev., 170, 401-478. [2] Moral, A. G. et al. (2018) LPSC 49, \#2449. [3] Wei, J. et al. (2015) J. Raman Spectrosc., 46, 810-821. [4] Abedin, M. N. et al. (2017) LPSC 47, \#1150. [5] Wiens, R. C. et al. (2017) LPSC 47, \#2600. [6] Tang, S. et al. (2016) Opt. Exp., 24(19), 22104-22109. [7] Beegle, L. W. et al. (2015) IEEE Aero. Con., 10.1109/AERO.2015.7119105. [8] Hu, F. et al. (2019) Nature Methods, 16, 830-842. [9] https://techport.nasa.gov/view/94431. [10] Tallarida, N. et al. (2018) LPSC 49, \#2779. [11] Acosta-Maeda, T. E. et al. (2019) LPSC 50, \#1713. [12] Viola, D., et al. (2018) LPSC 49, \#2083 [13] https://techport.nasa.gov/view/92282 [14] Murchie, S., et al. (2020) LPSC 51, \#1547. [15] Stone, W. et al. (2018) in: Outer Solar System, 429-541. [16] Li, X. et al. (2017) Int. J. Mass Spectrom., 422, 177-187. [17] Trainer, M.G. et al. (2018) LPSC 49, \#2586. [18] Niemann, H. B. et al. (2002) Space Sci. Rev., 104, 553-591. [19] Blase, R. et al. (2020) OPAG, \#6009. [20] Reh, K. et al. (2016) IEEE Aero. Con., 10.1109/AERO.2016.7500813. [21] Chua, E. J., (2016) Front. Mar. Sci., 3(209), 1-24. [22] Arevalo Jr, R., et al. (2019) AGU Fall, P34C-04. [23] Creamer, J. et al. (2020) OPAG, \#6005. [24] Mathies, R. A. et al. (2017) Astrobiology, 17(9), 902-912. [25] Golozar, M. et al. (2020) LPSC 51, \#2713. [26] Fernanda Mora, M. et al. (2019) ECS Meeting, \#2473. [27] Beckler, J. S. et al. (2014) Limnol. Oceanogr. Methods, 12(8), 563-576. [28] Southard, A. E. et al.,(2016) LPSC 47, \#2606. [29] Abrahamsson, V. et al. (2019) Anal.Bioanal. Chem., 411, 8091-8101. [30] Hecht, M. H. et al. (2009) Science, 325(5936), 64-67. [31] Noell, A. C., et al. (2019) AbSciCon, \#408-7. [32] Hecht, M. H., et al. (2008) JGR: Planets, 113. [33] Lindensmith, C. A. et al. (2016) PLoS one, 11(1), 1-23. [34] Serabyn, E. et al. (2016) Opt. Exp., 24(25), 28540-28548. [35] Mullen, A. D., et al. (2019) AbSciCon, \#482946. [36] Serabyn, G., et al. (2019) IEEE Aero. Con. 10.1109/

AERO.2019.8741627. [37] Bhartia, R. et al. (2010) App. Env. Microbiol. 76(21), 7231-7237. [38] Bibring, J.P. et. al. (2007) Space Sci. Rev., 128(1-4), 397-412 [39] Gaskin et. al. (2010), ASCE Earth \& Space, \#1246. [40] Lambert, J.L. et. al. (2010) AbSciCon, \#5657. [41] Carr., C. E. et al. (2017) IEEE Aero. Con., 10.1109/AERO.2017.7943896 [42] Scholin, C. A. et al. (2018) Oceanogr., 30(4), 100-113. [43] Srama, R., et al. (2004) Space Sci. Rev., 114, 65-518. [44] Kasprzak, W. K., et al. (1996) Proc. SPIE, 2803, 129-140. [45] Kempf, S., et al. (2014) EPSC, \#229. [46] Brockwell, T. G., et al. (2016) IEEE Aero. Con., 10.1109/AERO.2016.7500777. 